\title{
Boundary polarization in the six-vertex model
}

\author{
N. M. Bogoliubov, A. V. Kitaev, and M. B. Zvonarev \\ Steklov Institute of Mathematics at St. Petersburg, Fontanka 27, St. Petersburg 191011, Russia
}

(October 28, 2018)

\begin{abstract}
Vertical-arrow fluctuations near the boundaries in the six-vertex model on the two-dimensional $N \times N$ square lattice with the domain wall boundary conditions are considered. The one-point correlation function ("boundary polarization") is expressed via the partition function of the model on a sublattice. The partition function is represented in terms of standard objects in the theory of orthogonal polynomials. This representation is used to study the large $N$ limit: the presence of the boundary affects the macroscopic quantities of the model even in this limit. The logarithmic terms obtained are compared with predictions from conformal field theory.
\end{abstract}

PACS numbers: $05.50 .+\mathrm{q}, 05.70 . \mathrm{Np}, 02.30 . \mathrm{Ik}$

\section{THE MODEL}

In this paper, we shall consider the six-vertex model on a square lattice. Originally, this model was introduced as a model describing the ferroelectric properties of the hydrogen-bonded planar crystals [1]. The hydrogen atom positions are specified by attaching arrows to the lattice edges. In the six-vertex case, the arrows are arranged in such a way that there are always two arrows pointing away from, and two arrows pointing into, each lattice vertex (the so-called "ice rule"); thus, there are six possible states at each vertex (see, Fig. 1). The statistical weights, a, b and c, of the allowed states are invariant under the simultaneous reversal of all arrows:

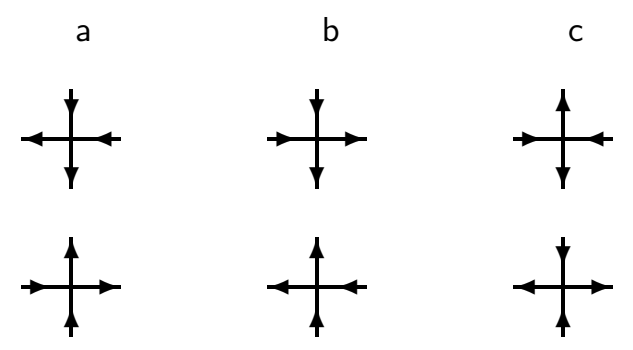

FIG. 1. The vertices of the six-vertex model and their statistical weights.

The partition function of the model on an $N \times N$ square lattice is obtained by summing over all possible arrow configurations $\{C\}$,

$$
Z_{N}=\sum_{\{C\}} \mathrm{a}^{n_{1}} \mathrm{~b}^{n_{2}} \mathrm{c}^{n_{3}}
$$

where $n_{1}, n_{2}$, and $n_{3}$ are the number of vertices of type a, $\mathrm{b}$, and $\mathrm{c}$ in configuration $C$, respectively $\left(n_{1}+n_{2}+n_{3}=\right.$ $N^{2}$.

The six-vertex model was studied for both periodic (PBC) [2, 3] and fixed boundary conditions [4]. In this paper, we are concerned exclusively with the domain wall boundary conditions (DWBC), namely, all arrows on the top and bottom of the lattice are pointing inward while all arrows on the left and right boundaries are pointing outward (see, Fig. 2).

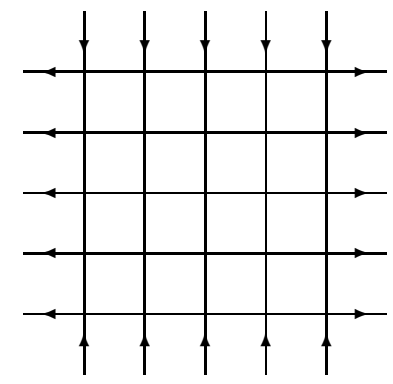

FIG. 2. The domain wall boundary conditions.

This model was introduced in Ref. [5] in connection with the calculation of the correlation functions for exactly solvable $1+1$ dimensional models [6]. It appears that some problems from the theory of alternating sign matrices [7,8] and domino tilings [9] may be reformulated in terms of this model.

Aperiodic boundary conditions are of interest since they demonstrate the influence of the boundaries and internal defects of real physical systems on their bulk properties. By renormalization group method, it was shown that the behavior of the correlation functions near the surfaces and defects is quite different from the bulk behavior [10]. The exactly solvable six-vertex model with the DW $\overrightarrow{\mathrm{BC}}$ provides us the opportunity to study the surface phenomena beyond renormalization group scheme.

\section{THE PARTITION FUNCTION AND THE BOUNDARY POLARIZATION}

The partition function $Z_{N}$ of the model with the DWBC may be represented as the determinant of an $N \times N$ matrix 11, 12. Though there exist several combinatorial representations for this determinant [13,14, it has so far only been calculated explicitly for some special cases [7]. Significant progress has recently been achieved 
in studying the asymptotics of $Z_{N}$ as $N \rightarrow \infty$. Under some special restrictions on the values of the vertex weights, the bulk free energy was calculated in Ref. 15] by using the Toda equation [16]. A more general result was obtained in Ref. [17] by a reformulation of the six-vertex model as a Hermitian matrix model to which saddle point integration method was applied. A representation convenient for the large $N$ analysis was suggested also in Ref. 18].

Less is known about the correlation functions of this model for at least two reasons. First, the calculation of the correlators, in general, is a more complicated problem than the calculation of the corresponding partition function. Second, the lack of translation invariance caused by the special boundary conditions introduces additional difficulties. Some correlation functions for the inhomogeneous model (the model with the statistical weights depending on the position of the vertex) with special choice of the weights were considered in Ref. [19].

The boundary polarization is the one-point correlation function that describes the probability for the arrow on the fixed lattice edge on the boundary to be pointing in either direction. The symmetry of the model allows us to consider only vertical arrows. Let us denote by $\chi_{N}$ the probability for the vertical arrow to be pointing down. Note that $\chi_{N}$ for PBC is independent of the position of the edge and is just a spontaneous polarization of the system [3]. Here, we shall discuss $\chi_{N}$ for the edge located at the lower-right corner of the lattice (in Fig. 3 this edge is dotted):

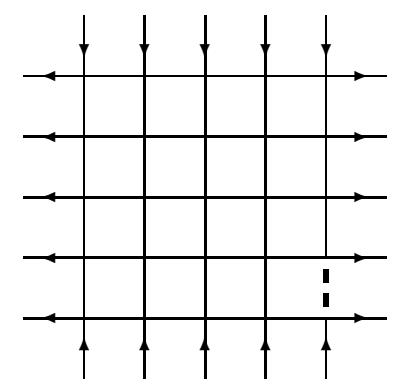

FIG. 3. $\chi_{N}$ is calculated for the arrow on the dotted edge.

It turns out that if the arrow on the dotted edge is pointing down, then due to the imposed boundary conditions, the allowed vertex configuration at the bottom and the right boundaries is determined in a unique way. Actually, in the lower-right corner one has the vertex of type c; thus, the rest of the $2 N-2$ vertices are of type b, and the DWBC are valid for the residual $N-1 \times N-1$ sublattice. Thus,

$$
\chi_{N}=\mathrm{cb}^{2 N-2} \frac{Z_{N-1}}{Z_{N}}
$$

and the problem of studying the correlation function, $\chi_{N}$, is reduced to the analysis of the ratio of the partition functions $Z_{N-1} / Z_{N}$.
The boundary polarization on the arbitrary edge is expressed in terms of linear combination of partition functions on sublattices.

\section{THE CONNECTION WITH ORTHOGONAL POLYNOMIALS}

Due to the multiplicativity of the partition function, one can set $c=1$ without loss of generality. Hence, the model is characterized by only two parameters, a and b. In Fig. 4 the phase diagram on the $(\mathrm{a}, \mathrm{b})$ plane for the model with PBC is plotted (cf., Fig. 8.5 of Ref. [3]). It may be regarded as the phase diagram for the model with the DWBC, in the sense that the free energy takes a different analytic form in the regions divided by the solid and dashed lines (see Ref. 17] for details). Naturally, the ground state and low temperature behavior do not coincide with those for the model with PBC.

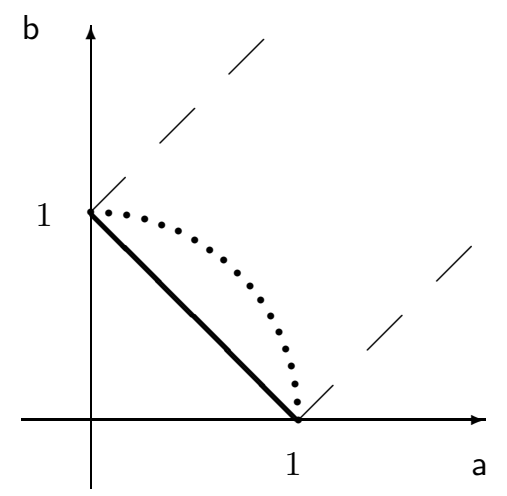
b.

FIG. 4. The phase diagram in terms of the weights a and

In this and next section, we shall consider the model on the solid line in Fig. A. It is convenient to use the following parametrization of the vertex weights on this line:

$$
\left\{\begin{array}{l}
\mathrm{a}=1 / 2-x \\
\mathrm{~b}=1 / 2+x
\end{array}, \quad-1 / 2<x<1 / 2\right.
$$

It follows from Ref. 11] that the partition function of the model is represented as the determinant of an $N \times N$ matrix:

$$
Z_{N}=\frac{\operatorname{det}_{N} \mathcal{M}}{[\phi(x)]^{N^{2}}\left(\prod_{k=1}^{N-1} k !\right)^{2}}
$$

where the matrix elements of $\mathcal{M}$ are given by

$$
\mathcal{M}_{\alpha k}=\frac{d^{\alpha+k}}{d x^{\alpha+k}} \phi(x), \quad \alpha, k=0,1, \ldots, N-1
$$

and $\phi(x)=\left[\left(\frac{1}{2}-x\right)\left(\frac{1}{2}+x\right)\right]^{-1}$. 
Matrix $\mathcal{M}$ is a Hankel matrix, that is, it has constant entries along the antidiagonals. Its determinant can be expressed in terms of objects related to the theory of orthogonal polynomials. Let $p_{n}(\xi), n \geq 0$, be a sequence of monic orthogonal polynomials with weight $\rho(\xi)$ :

$$
\int_{-\infty}^{\infty} p_{n}(\xi) p_{m}(\xi) \rho(\xi) d \xi=\delta_{m n} h_{n}, \quad n, m \geq 0 .
$$

Then the determinant of the Hankel matrix with the elements

$$
\mathcal{H}_{\alpha k}=\int_{-\infty}^{\infty} \xi^{\alpha+k} \rho(\xi) d \xi, \quad \alpha, k=0,1, \ldots, N-1
$$

is equal to 14 $\operatorname{det}_{N} \mathcal{H}=h_{0}^{N} b_{1}^{N-1} b_{2}^{N-2} \cdots b_{N-2}^{2} b_{N-1}$. Here $h_{0}$ is determined from Eq. (4), and $b_{n}$ are the coefficients of the corresponding three-term recurrence relation,

$$
p_{n+1}(\xi)=\left(a_{n}+\xi\right) p_{n}(\xi)-b_{n} p_{n-1}(\xi), \quad n \geq 1,
$$

with the initial conditions $p_{0}(\xi)=1$ and $p_{1}(\xi)=\xi+a_{0}$.

The function $\phi(x)$ has the following integral representation:

$$
\phi(x)=\int_{-\infty}^{\infty} e^{-x \xi} e^{-|\xi| / 2} d \xi
$$

Combining this representation with Eq. (3) and Eq. (5), one shows that $\operatorname{det}_{N} \mathcal{M}=\operatorname{det}_{N} \mathcal{H}$, where the weight $\rho(\xi)$ is equal to

$$
\rho(\xi)=e^{-x \xi} e^{-|\xi| / 2}
$$

Then, since the coefficients $b_{n}$ and $h_{n}$ satisfy the wellknown relation $b_{n}=h_{n} / h_{n-1}, n \geq 1$, we have $\operatorname{det}_{N} \mathcal{M}=$ $h_{0} h_{1} \cdots h_{N-1}$, and the desired representation for probability (11) is 20

$$
\chi_{N+1}=\mathrm{a}^{-2 N-1} \mathrm{~b}^{-1} \frac{(N !)^{2}}{h_{N}} .
$$

Now let us discuss briefly the general case when a and $\mathrm{b}$ are arbitrary positive constants. The partition function is given by formulas (2) and (3), while $\phi(x)$ should be changed (see, for example, [6,11,12]), the weight $\rho(\xi)$ is obtained by straightforward calculations [17], and the corresponding expression for $\chi_{N+1}$ differs from Eq. (7) by a constant.

\section{THE LARGE $N$ LIMIT}

Equation (7) reduces the problem of calculation of the probability $\chi_{N+1}$ to the calculation of the normalizing coefficient $h_{N}$. At present there exists a powerful method for studying the $N \rightarrow \infty$ behavior of $h_{N}$. This method is based on the matrix Riemann-Hilbert conjugation problem 21. The corresponding asymptotic technique was suggested in Ref. 22] and worked out for orthogonal polynomials with nonanalytic weights in Ref. [23]. Using this technique, we obtain the main result of the paper:

$$
\begin{aligned}
& \ln h_{N}=2 N \ln N+2 N \ln \left[\frac{\pi e^{-1}}{\cos (\pi x)}\right]+\ln N \\
& +\ln \left[\frac{2 \pi^{2}}{\cos (\pi x)}\right]+\frac{1}{4 N}+\frac{\varphi(x, N)}{2 N(\ln N)^{2}}+\cdots,
\end{aligned}
$$

where

$$
\varphi(x, N)=(-1)^{N} \cos [2 \pi x(N+1 / 2)]
$$

and the omitted terms are of the order $1 /\left[N(\ln N)^{3}\right]$. Note that for $x=0$ this result coincides with the one obtained in Ref. 23.

Having expansion (8), it is easy to find the expansion for $\chi_{N}$ :

$$
\begin{aligned}
& \ln \chi_{N}= \\
& -2 N \ln \left[\frac{\pi(1 / 2-x)}{\cos (\pi x)}\right]+\ln \left[\frac{\pi}{\cos (\pi x)} \frac{1 / 2-x}{1 / 2+x}\right] \\
& -\frac{1}{12 N}-\frac{\varphi(x, N-1)}{2 N(\ln N)^{2}}+\ldots
\end{aligned}
$$

From Eq. (9), we get all increasing terms for the partition function $Z_{N}$,

$$
Z_{N} \underset{N \rightarrow \infty}{\sim} C \exp \left(f_{0} N^{2}+f_{1} N+f_{2} \ln N\right)
$$

where $C>0$ is a bounded function of $N$, and

$$
f_{0}=\ln \left[\frac{\pi\left(1 / 4-x^{2}\right)}{\cos (\pi x)}\right], \quad f_{1}=0, \quad f_{2}=\frac{1}{12} .
$$

\section{RESULTS AT OTHER POINTS}

At present there are several points on the phase diagram, where the determinant has been calculated in closed form.

(i) The "free-fermion case", $a^{2}+b^{2}=1$ (dotted circular quadrant on the phase diagram). On this circle, one has a very simple result $Z_{N}=1$. This result can be obtained by an appropriate limit from the inhomogeneous partition function 6, 12]. Therefore, one has

$$
\ln \chi_{N}=2(N-1) \ln \mathrm{b} .
$$

(ii) The "ice point", $a=b=1$. All weights are equal, and the partition function is just the number of allowed configurations $\{C\}$ (Sec. I). In this case one has Ref. [7]

$$
Z_{N}=\prod_{j=1}^{N} \frac{(3 j-2) !}{(N-1+j) !}
$$

thus, 


$$
\ln \chi_{N}=N \ln \frac{16}{27}-\frac{1}{2} \ln \frac{16}{27}+\frac{5}{36} \frac{1}{N}+\frac{5}{72} \frac{1}{N^{2}}+\cdots .
$$

For $Z_{N}$ we have asymptotic expansion (10), where $f_{0}=$ $\ln \frac{3 \sqrt{3}}{4}, f_{1}=0$ and $f_{2}=-\frac{5}{36}$. Note that for the model with PBC [3], $f_{0}^{\mathrm{PBC}}=\ln \frac{8}{3 \sqrt{3}}>f_{0}$, that is, for large $N$ the number of allowed configurations for the DWBC is less than for periodic ones.

(iii) The point $\mathrm{a}=\mathrm{b}=1 / \sqrt{3}$. In this case 7

$$
\begin{aligned}
& Z_{2 N+1}=\frac{1}{3^{N^{2}}}\left[\prod_{j=1}^{N} \frac{(3 j-1) !}{(N+j) !}\right]^{2} \\
& Z_{2 N}=\frac{(3 N-1) !(N-1) !}{3^{N}[(2 N-1) !]^{2}} Z_{2 N-1},
\end{aligned}
$$

and we immediately get

$$
\ln \chi_{N}=N \ln \frac{4}{9}+\frac{1}{2} \ln \frac{27}{4}-\frac{1}{18} \frac{1}{N}+\cdots ;
$$

thus, $f_{0}=\ln \frac{\sqrt{3}}{2}, f_{1}=0$ and $f_{2}=\frac{1}{18}$. The first omitted term in Eq. (13) is of the order $N^{-2}$, and depends on the parity of $N$.

We complete this paper with the following statement. One can see that in expansion (9), which was obtained for $a+b=1$, there exists a logarithmic term. For arbitrary a and $\mathrm{b}$, we state that the logarithmic terms in the large $N$ expansion of $\ln \chi_{N}$ will take place only under the following conditions: weights $\mathrm{a}$ and $\mathrm{b}$ are either on the solid line $a+b=1$, or on the dashed lines $|a-b|=1$ of the phase diagram (Fig. (1). For the model with PBC, the logarithmic terms could be explained via conformal field theory [6]. Similar arguments are valid for the discussed model. The influence of the boundary condition changing operators 24] should also now be taken into consideration. If we move away from the solid or dashed lines, the logarithmic terms vanish and the expansion of $\ln \chi_{N}$ will be in integer powers of $N$. Expansions (11), (12) and (13) indeed contain only integer powers of $N$, thus confirming the above statement.

Finally, we would like to mention that the six-vertex model with any boundary conditions can be considered as a model for a description of interface roughening of a crystal surface [25]. An important point in these studies is the existence of exact analytical results, which are known for the six-vertex model with PBC [1] 3]. We believe that our analytical results for the model with DWBC provide one more basis for the experiments and simulations in this direction.

We thank A. H. Vartanian for bringing Ref. [23] to our attention. This work was partially supported by the RFBR Grant No. 01-01-01045.
[1] E.H. Lieb, F.Y. Wu, in Phase Transitions and Critical Phenomena, edited by C. Domb and M.S. Green (Academic Press, London, 1972), Vol. 1, p. 321.

[2] E.H. Lieb, Phys. Rev. 162, 162 (1967); Phys. Rev. Lett. 18, 1046 (1967); Phys. Rev. Lett. 19, 108 (1967); B. Sutherland, Phys. Rev. Lett. 19, 103 (1967).

[3] R.G. Baxter, Exactly Solved Models in Statistical Mechanics (Academic press, San Diego, 1982).

[4] R.J. Baxter and A.L. Owczarek, J. Phys. A 22, 1141 (1989); M.T. Batchelor, R.J. Baxter, M.J. O'Rourke, and C.M. Yung, J. Phys. A 28, 2759 (1995); K. Eloranta, J. Stat. Phys. 96, 1091 (1999); C.B. Thorn, Phys. Rev. D 63, 105009 (2001).

[5] V.E. Korepin, Commun. Math. Phys. 86, 391 (1982).

[6] V.E. Korepin, N.M. Bogoliubov, and A.G. Izergin, Quantum Inverse Scattering Method and Correlation Functions (Cambridge University Press, Cambridge, 1993).

[7] G. Kuperberg, Int. Math. Res. Notices 3, 139 (1996).

[8] D.M. Bressoud, Proofs and Confirmations; The Story of the Alternating Sign Matrix Conjecture (Cambridge University Press, Cambridge, 1999).

[9] H. Cohn, N. Elkies, and J. Propp, Duke Math. J. 85, 117 (1996); W. Jockush, J. Propp, and P. Shor, e-print math.CO/9801068.

[10] A. Hanke and M. Kardar, Phys. Rev. Lett. 86, 4596 (2001); A. Hanke, Phys. Rev. Lett. 84, 2180 (2000); A. Hanke, M. Krech, F. Schlesener, and S. Dietrich, Phys. Rev. E 60, 5163 (1999).

[11] A.G. Izergin, Dokl. Akad. Nauk (SSSR) 297, 331 (1987) [Sov. Phys. Dokl. 32, 878 (1987)].

[12] A.G. Izergin, D.A. Coker, and V.E. Korepin, J. Phys. A 25, 4315 (1992).

[13] A. Lascoux, Séminaire Lotharingien Combin. 42 Art. B42p, 15pp. (1999).

[14] C. Krattenthaler, Séminaire Lotharingien Combin. 42 Art. B42q, 67pp. (1999).

[15] V.E. Korepin and P. Zinn-Justin, J. Phys. A 33, 7053 (2000).

[16] K. Sogo, J. Phys. Soc. Jpn. 62, 1887 (1993); A.G. Izergin, E. Karjalainen, N.A. Kitanine, J. Math. Sci. (New York) 100, 2141 (2000).

[17] P. Zinn-Justin, Phys. Rev. E 62, 3411 (2000).

[18] N.A. Slavnov, Zap. Nauchn. Sem. POMI 269, 308 (2000) (available at cond-mat/0005298).

[19] V. Korepin and P. Zinn-Justin, e-print nlin.SI/0008030; J. de Gier and V. Korepin, e-print math-ph/0101036.

[20] N.M. Bogoliubov, A.V. Kitaev, and M.B. Zvonarev, Zap. Nauchn. Sem. POMI 269, 136 (2000).

[21] A.S. Fokas, A.R. Its, and A.V. Kitaev, Commun. Math. Phys. 142, 313 (1991); 147, 395 (1992).

[22] P. Deift, T. Kriecherbauer, K. T-R McLaughlin, S. Venakides, and X. Zhou, Int. Math. Res. Notices 16, 759 (1997); Commun. Pure Appl. Math. 52, 1491 (1999).

[23] T. Kriecherbauer and K. T-R McLaughlin, Int. Math. Res. Notices 6, 299 (1999).

[24] J.L. Cardy, Nucl. Phys. B 342, 581 (1989).

[25] H. van Beijeren, Phys. Rev. Lett. 38, 993 (1977); E. Carlon, G. Mazzeo, and H. van Beijeren, Phys. Rev. B 55, 757 (1997); 